\title{
Evaluación de validez convergente y discriminante en tests computarizados de toma de decisiones ${ }^{1}$
}

\author{
Ignacio Acuña², Yanina Michelini, Juan Ignacio Guzmán, Juan Carlos Godoy \\ Insituto de Investigaciones Psicológicas, UNC-CONICET, Córdoba, Argentina
}

\section{RESUMEN}

El objetivo de este trabajo fue analizar la validez convergente y discriminante entre tests de impulsividad, funcionamiento ejecutivo, toma de decisiones (TD) con y sin componente emocional y de TD centrado en el agente. Mediante un diseño instrumental, se evaluaron 217 participantes (64,5\% mujeres) entre 18 y 30 años de edad $(M=23,22, \pm 2,44)$. El Análisis de Componentes Principales mostró 4 componentes que explicaron el $83,88 \%$ de la varianza y agruparon los tests de acuerdo a lo esperado. Además, el estudio de las correlaciones mostró que los tests de TD verídica fueron levemente correlacionados, sugiriendo convergencia parcial entre ellas. No se encontraron correlaciones significativas entre los tests de TD verídica y los tests de TD centrados en el agente, función ejecutiva e impulsividad. Los resultados coincidieron con las expectativas y con los estudios previos sobre el tema.

Palabras clave: Toma de Decisiones; Impulsividad; Funcionamiento Ejecutivo; Validez del test, Análisis de Componentes Principales.

RESUMO - Avaliação da Validade Convergente e Discriminante em Testes Computadorizados da Tomada de Decisões O objetivo deste estudo foi analisar a validade convergente e discriminante entre testes de impulsividade, de funcionamento executivo, de tomada de decisão (TD) com e sem componente emocional e de TD focada no agente. Através de um desenho Instrumental, foram avaliados 217 participantes (64,5\% mulheres) entre 18 e 30 anos $(M=23,22 \pm 2,44)$. A Análise de Componentes Principais mostrou quatro componentes que explicaram $83,88 \%$ de variância e agrupo os testes como foi esperado. Além disso, o estudo das correlações mostra que os testes da TD verdadeira foram fracamente correlacionados, sugerindo convergência parcial entre elas. Não foram encontradas correlações significativas entre os testes da TD verdadeira e os testes da TD com foco no agente, função executiva e impulsividade. Os resultados coincidem com as expectativas e com estudos prévios sobre o assunto.

Palavras-chave: Tomada de Decisão; Impulsividade; Funcionamento Executivo; Validade do teste; Análise de Componentes Principais.

\section{ABSTRACT - Assessment of convergent and discriminant validity in computerized decision-making tests}

The objective of this study was to analyze the convergent and discriminant validity between impulsivity, executive functioning, and decision-making (DM) tests with and without emotional component and and agent centered DM. Through an Instrumental design, 217 participants were evaluated, 64.5\% women, between 18 and 30 years of age $(M=23.22 \pm 2.44)$. The Principal Component Analysis showed four components that explained $83.88 \%$ of variance and grouped the tests as expected. In addition, the correlation study shows that the true DM tests were poorly correlated, suggesting partial convergence between them. No significant correlations were found between true DM testing and DM testing with agent focus, executive function and impulsivity. The results coincide with expectations and previous studies on the subject.

Keywords: Decision-making; Impulsivity; Executive functioning; Test validity; Principal component analysis.

\section{Introducción}

Al momento de incluir un instrumento en una batería de evaluación neurocognitiva es necesario considerar sus propiedades. Dos de las propiedades más importantes sobre las que hay que atender en una prueba psicológica son la población para la que fue pensada y la validez de criterio de la prueba en cuestión (Hogan, 2004).
En relación a la población para la cual fue pensada la prueba, es necesario tener en cuenta las diferencias que se pueden encontrar entre la población origen de los instrumentos que se planea estudiar y la población en la que se desea aplicarlos. Cuando se evalúan valores personales, rasgos de personalidad, se espera que estos constructos varíen de individuo a individuo, y por tanto, en la sociedad. De hecho, recientemente se ha puesto en duda la noción que diversos dominios cognitivos

El presente trabajo se realizó con el aporte del Consejo Nacional de Investigaciones Científicas y Técnicas (CONICET), Argentina, mediante una Beca Doctoral al primer autor, y un subsidio para investigación de la Secretaría de Ciencia y Técnica de la Universidad Nacional de Córdoba.

Endereço para correspondência: Instituto de Investigaciones Psicológicas (IIPSI-UNC-CONICET), Facultad de Psicología, Universidad Nacional de Córdoba. Enrique Barros esq. Enfermera Gordillo, Estafeta Postal 32, Ciudad Universitaria, Código Postal 5000, Córdoba, Argentina. Tel.: (+54) 351-433-4064, Int. 164 E-mail: ignacio.acuna@unc.edu.ar 
sean de carácter universal. Mayor es la importancia de las diferencias poblacionales cuando se trata de constructos asociados al desempeño neurocognitivo como la toma de decisiones y la función cognitiva, de los cuales no se tiene información respecto de cómo varían en función del contexto socio-cultural. (Véase: Crivelli, Jarillo, Russell, \& Fernández-Dols, 2016; Henrich, Heine, \& Norenzayan, 2010).

La validez de criterio consiste en establecer un vínculo entre el desempeño en la prueba que estamos considerando, y el desempeño en algún otro criterio que se toma como un indicador importante del constructo (American Educational Research Association, American Psychological Association, \& National Council on Measurement in Education, 2014; Hogan, 2004). Una de las aproximaciones para establecer la validez de criterio es comparar el puntaje de la prueba evaluada con otra prueba (Carretero-Dios \& Pérez, 2007; Hogan, 2004). En este caso, adquieren importancia los conceptos de validez convergente y validez discriminante. La primera entendida como la correlación positiva entre las pruebas consideradas, lo que indicaría que ambas pruebas reclutan el mismo proceso. Y la segunda, entendida como la ausencia de correlaciones estadísticas entre las puntuaciones de las pruebas, lo que indicaría que las pruebas en cuestión no involucran los mismos procesos (Hogan, 2004).

La Toma de Decisiones (TD) se define como la capacidad para seleccionar un curso de acción entre un conjunto de posibles alternativas conductuales. Se trata de un proceso complejo en el que intervienen diversas claves ambientales, los aspectos cognitivos de la situación de decisión, las contingencias de recompensa y castigo asociadas a cada una de las opciones, y las señales emocionales asociadas a cada una de las posibles respuestas (Martínez-Selva, Sánchez-Navarro, Bechara, \& Román, 2006; Reimann \& Bechara, 2010).

En el campo de la evaluación neuropsicológica, la evaluación de la TD suele dividirse en dos escenarios posibles: bajo condiciones de riesgo ambiguo y bajo condiciones de riesgo objetivo (Schiebener \& Brand, 2015). En el primero, quien decide no recibe ningún tipo de información sobre los posibles resultados o las probabilidades de éxito y fracaso asociados a cada opción disponible. Es decir, las reglas del juego son implícitas, y la decisión se basa principalmente en la consideración, consciente o no, sobre las consecuencias de decisiones similares en el pasado. En el segundo, los posibles resultados y probabilidades de éxito y fracaso pueden ser calculados, al menos en parte. Es decir, las reglas del juego están explícitas y la decisión puede ser tomada en función de los resultados esperados (Schiebener \& Brand, 2015).

Los procesos de TD involucrados en el contexto de riesgo ambiguo pueden ser explicados desde la propuesta de la Hipótesis del Marcador Somático (HMS). En este contexto la TD es guiada por la emoción, considerada como reacciones subjetivas y somáticas, que pueden presentarse en forma de cambios vegetativos, musculares, neuroendocrinos o neurofisiológicos, ante ciertos acontecimientos, positivos o negativos y que pueden ser conscientes o no (Martínez-Selva et al., 2006; Reimann \& Bechara, 2010). Cuando el individuo se enfrenta a una situación de decisión novedosa, su elección conlleva una consecuencia emocional reforzante o aversiva, disparada por la amígdala. Una vez que esta reacción emocional ha sido experimentada al menos una vez, la corteza prefrontal ventromedial será la encargada de vincularla con la opción elegida. De esta manera la situación de decisión queda marcada somáticamente (emocionalmente). Cuando el individuo se enfrente a una situación de decisión igual o similar a la ya experimentada, la corteza prefrontal recuperará el "marcador somático" y el proceso de toma de decisiones será mucho más eficiente y eficaz ya que se evocará las emociones experimentadas ante la decisión anterior. Así, por ejemplo, si la decisión tuvo consecuencias negativas, el individuo debería evitar las opciones que llevaron a experimentar ese tipo de emoción (Reimann \& Bechara, 2010). La evaluación de la capacidad de tomar decisiones en este tipo de contextos ha sido clásicamente evaluada utilizando la Iowa Gambling Task (Bechara, Damasio, Damasio, \& Anderson, 1994).

Por otro lado, los procesos de TD involucrados en escenarios de riesgo objetivo, presentan otro tipo de explicación. En efecto, en el modelo original (Brand, Recknor, Grabenhorst, \& Bechara, 2007; Schiebener \& Brand, 2015) se propone que si hay información disponible sobre el riesgo que conlleva una decisión, resultaría adaptativo controlar dicho riesgo cognitivamente durante la TD, ya sea calculando las probabilidades, comparando las alternativas o advirtiendo posibles consecuencias de cada opción. Este tipo de control cognitivo resulta particularmente útil en contextos de TD con riesgo objetivo. Así, en consonancia con su propuesta, Brand y colaboradores (2005) desarrollaron una tarea de evaluación de toma de decisiones, la Game of Dice Task, con reglas estables y explícitas, haciendo posible que los participantes se esfuercen en obtener ganancias y evitar pérdidas aplicando estrategias y reglas de razonamiento.

Por otro lado, de acuerdo con Podell, Funk, \& Goldberg, (2012), una manera óptima de estudiar la TD es utilizando paradigmas cognitivos que permitan realizar elecciones sin restricciones, que no tengan clasificaciones a priori sobre la calidad de las respuestas. Este tipo de paradigmas deberían apuntar a estudiar las preferencias subjetivas de quien decide antes que su capacidad para realizar decisiones correctas o incorrectas. Así, la distinción entre la cognición "verídica" y la cognición "centrada en el agente" es central para comprender la naturaleza de la toma de decisiones. Estos autores entienden a la TD "verídica" como aquella que implica valoraciones cualitativas sobre la decisión (correcto vs. incorrecto). La TD "centrada en el agente", por su parte, es aquella que 
carece de estas valoraciones a priori y centra su análisis en las características subjetivas de quien decide. La primera estará destinada a la resolución de problemas, mientras que la segunda abarcará decisiones que pueden ser triviales (por ejemplo, ¿qué bebida ingerir en la próxima comida?) o de mucho impacto en la vida de quien decide (por ejemplo, ¿qué carrera estudiar?). En este sentido, se ha sugerido que la corteza prefrontal está implicada en el proceso de tomar decisiones centradas en el agente ya que son fundamentales para formar las representaciones subjetivas del mundo y, consecuentemente, para fijar los planes y objetivos que guiarán al individuo (Goldberg \& Podell, 2000). Consecuentemente, Goldberg, Harner, Lovell, Podell, \& Riggio, (1994) proponen un paradigma experimental para evaluar la toma de decisiones centrada en el agente, donde no se prioriza establecer la cualidad (correcto/incorrecto) de las respuestas sino su "estilo". Así, mediante la tarea Cognitive Bias Task, se busca determinar si las decisiones tomadas por los participantes tienen un estilo "independiente del contexto" o, por el contrario, uno "dependiente del contexto" (VerdejoGarcía et al., 2006).

Entonces, tomando en consideración la literatura revisada, este estudio tuvo como objetivo principal analizar la validez convergente y discriminante de un conjunto de pruebas conductuales diseñadas para evaluar la impulsividad rasgo (Escala UPPS-P), la función ejecutiva (Wisconsin Card Sorting Test), la TD verídica con y sin componente emocional (Iowa Gambling Task y Game of Dice Task, respectivamente) y la TD centrada en el agente (Cognitive Bias Task). Al respecto, hipotetizamos que las pruebas de TD verídica presentarán validez convergente entre ellas y que, a su vez, presentarán validez discriminante con las pruebas de TD centrada en el agente, y con las de función ejecutiva y de impulsividad rasgo.

\section{Método}

\section{Diseño}

Se utilizó un diseño Instrumental (Montero \& León, 2007) a través del cual se evaluó la validez convergente y discriminante de pruebas de TD. Para ello se siguieron las recomendaciones de Carretero-Dios \& Pérez, (2007) y Muñiz, Elosua, \& Hambleton, (2013), así como las recomendaciones de la American Educational Research Association, y de la American Psychological Association National y del Council on Measurement in Education (American Educational Research Association et al., 2014).

\section{Participantes}

La muestra inicial estuvo compuesta por 269 participantes, se eliminaron 50 casos por registros de información incompletos y erróneos. La muestra final estuvo compuesta por 217 participantes voluntarios de ambos sexos (64,5\% mujeres) entre 18 y 30 años de edad $(M=23,22, D S \pm 2,44)$, que reportaron tener entre 12 y 18 años de educación formal $(M=14,60, \mathrm{DS} \pm, 110)$. La convocatoria fue realizada a través redes sociales y en las aulas de la Universidad Nacional de Córdoba (UNC). Los participantes debieron cumplir los siguientes requisitos: no tener, ni haber tenido, alguna alteración neurológica o psiquiátrica ni estar bajo tratamiento por dichas afecciones, no poseer alguna adicción o estar bajo tratamiento por adicción. Esta información se relevó mediante autoreporte (Wood, Sher, \& Rutledge, 2007). Además, se les informó que podían abandonar el estudio en cualquier momento y se les aseguró que los datos recabados solo se utilizarían con fines estadísticos y permanecerían en anonimato.

\section{Instrumentos}

Cuestionario sociodemográfico: se incluyeron preguntas sobre edad, sexo, nivel educativo, estado civil, historia familiar de consumo, padecimiento de enfermedades y/o tratamientos.

Wisconsin Card Sorting Test (WCST; Grant \& Berg, 1948): esta prueba evalúa funciones ejecutivas. Se utilizó la versión electrónica de 128 cartas. Requiere que el participante empareje las cartas que aparecen en el mazo de abajo, con uno de las cuatro mazos que aparecen arriba, haciendo clic debajo de la carta con la que cree que coincide. Cuando el participante realiza diez clasificaciones correctas consecutivas, consigue una categoría y se cambia el criterio de clasificación sin previa advertencia. Además, se le menciona que se le señalará si lo hizo de manera correcta o incorrecta y que, de acuerdo con esa información, debe tratar de obtener tantas cartas correctas como sea posible. El participante debe clasificar primero por color, luego por forma y luego por número. El procedimiento continúa hasta que se han completado 6 categorías correctas o hasta que se han colocado las 128 cartas (Heaton, Chelune, Talley, Kay, \& Curtis, 1993).

Iowa Gambling Task (IGT; Bechara, Damasio, Damasio, \& Anderson, 1994): esta prueba evalúa la habilidad para tomar decisiones ventajosas y desventajosas bajo riesgo ambiguo. Requiere que el individuo seleccione entre diversas opciones asociadas a contingencias ambiguas de recompensa y castigo. Se presentan cuatro mazos de cartas (A, B, C y D). El individuo tiene que elegir cartas de estos cuatro mazos durante 100 ensayos. El número de cartas que contiene cada mazo y el número total de ensayos son desconocidos para el individuo, al que se le pide que decida en cada ensayo hasta que finalice el juego, con el objetivo de intentar ganar el máximo dinero posible. Cada una de las cartas está asociada a una recompensa monetaria inmediata, y en algunos casos también se asocian a castigos (en forma de pérdida monetaria). Aunque es un factor que el participante desconoce, los mazos A y B son desventajosos, ya que generan mayores recompensas inmediatas pero 
producen pérdidas más severas y llevan a un resultado negativo a largo plazo. En cambio, los mazos C y D son ventajosos ya que proporcionan recompensas menores, pero también producen castigos menos severos, generando beneficios a largo plazo. Por lo tanto, para conseguir ganar dinero en la tarea, la estrategia apropiada es seleccionar de manera consistente más cartas de los mazos ventajosos (C y D), que de los desventajosos (A y B) (Reimann \& Bechara, 2010). Para puntuar el rendimiento del participante en la tarea, se suman el total de cartas elegidas de los mazos desventajosos (A y B) y se resta el resultado de esta suma al total de cartas elegidas de los mazos ventajosos (C y D), el resultado de esta suma da un puntaje neto para el total de la prueba.

Game of Dice Task (GDT; Brand et al., 2005): es una tarea diseñada para medir la influencia de la función ejecutiva en la toma de decisiones en contextos de riesgo objetivo. A los participantes se les solicita que incrementen su capital inicial $(\$ 1.000)$ dentro de los $\mathrm{N}$ tiros del dado. Antes de cada tiro, los participantes tienen que elegir un solo número o combinación de números (dos, tres, o cuatro números). Cada opción está relacionada con una ganancia y una pérdida ficticia que dependen de la probabilidad de ocurrencia de la opción (Un solo número: $\$ 1.000$ de ganancia/pérdida; la combinación de dos números: \$500 de ganancia/pérdida; la combinación de tres números $\$ 200$ de ganancia/pérdida; la combinación de cuatro números: $\$ 100$ de ganancia/pérdida). las reglas y el grado de ganancias y pérdidas y pérdidas son descriptos explícitamente y visualizadas. A los participantes también se les informa que tienen que realizar un total de 30 decisiones. Antes de cada tiro, la ganancia -en caso de congruencia entre el/los números/s seleccionado/s y el número tirado- o pérdida -en caso de incongruencia entre el/los número/s seleccionado/s y el número tiradoy el capital modificado son presentados visualmente y señalados por dos señales acústicas diferentes (ganancia: tintineo de una caja registradora; pérdida: un sonido sordo). Además, el número de tiros remanentes es también mostrado en la pantalla. El resultado de los tiros es pseudo-aleatorizado, lo que permite que cada uno de los seis posibles números salga $\mathrm{N}$ veces durante la tarea, pero en un orden balanceado (Brand et al., 2007). Para realizar las puntuaciones, se clasifican las elecciones de uno y dos números (con probabilidades de ganar menores al 50\% y altas ganancias pero también altas penalidades) como riesgosa o desventajosa, respectivamente, mientras que las opciones de tres y cuatro números (con probabilidades de ganar del $50 \%$ y superiores, bajas ganancias pero también bajas penalidades) como no riesgosa y ventajosa, respectivamente. Al total de elecciones de tres y cuatro números se le resta el total de elecciones de uno y dos números (Brand et al., 2007). La fórmula es la siguiente: Puntaje neto $=($ Elecciones ventajosas + Elecciones No riesgosas) - (Elecciones Desventajosas + Elecciones Riesgosas).
Cognitive Bias Task (CBT; Goldberg, Harner, Lovell, Podell, \& Riggio, 1994): es una prueba que evalúa estilo de toma de decisiones mediante un paradigma de selección de respuestas de múltiple opción. Consiste en la presentación de estímulos caracterizados en cinco dimensiones: color (rojo/azul), forma (círculo/cuadrado), número (uno/dos), tamaño (grande/pequeño) y contorno (delimitado/relleno con un color homogéneo). Dos estímulos cualesquiera pueden ser comparados en función del número de dimensiones que compartan. Por cada ensayo, primero aparece la carta de arriba y luego dos cartas más, abajo, entre las cuales el participante debe elegir la que más prefiere. Para cada elección se computa un índice de similitud que va de 0 a 5. Los índices de similitud de cada elección se suman a lo largo de la prueba, generando una puntuación que va de 80 a 220 puntos. Esto último representa el puntaje total de la prueba. En ambos casos, puntajes extremos (ya sean bajos o altos) significan que el participante hizo elecciones dependientes del contexto cognitivo, ya sea elecciones sistemáticamente más semejantes (puntajes altos) o más diferentes (puntajes bajos); y puntajes medios (alrededor de 150 puntos) significan que el participante hizo elecciones independientes del contexto cognitivo. Las elecciones independientes del contexto, son decisiones tomadas en base a las representaciones preexistentes de la persona e implican un intento por satisfacer las necesidades de acuerdo a estas representaciones preexistentes sin considerar las condiciones del ambiente ni de la situación de decisión. Por otro lado, las decisiones dependientes del contexto intentan capturar las características únicas y específicas de la situación de decisión y así flexibilizar y adaptar las representaciones previas considerando las características de la situación, y así seleccionar la mejor opción posible.

Escala de impulsividad UPPS-P (Verdejo-García, Lozano, Moya, Alcázar, \& Pérez-García, 2010): Se utilizó la adaptación española de esta escala. La escala es un inventario de 59 ítems diseñado para medir cinco aspectos distintos de la personalidad que llevan a la conducta impulsiva: la urgencia negativa, la (falta de) perseverancia, la (falta de) premeditación, la búsqueda de sensaciones, y la urgencia positiva. La primera subescala, la urgencia negativa, evalúa la tendencia del individuo a ceder a los impulsos fuertes, especialmente cuando se acompañan de emociones negativas como la depresión, la ansiedad o la ira. La segunda subescala, la (falta de) perseverancia, evalúa la capacidad del individuo para persistir en la realización de trabajos o de las obligaciones a pesar de aburrimiento y/o fatiga. La tercera subescala, la (falta de) premeditación, evalúa la capacidad del individuo para pensar en las posibles consecuencias de su comportamiento antes de actuar. La subescala número cuatro, la búsqueda de sensaciones, evalúa la preferencia de la persona para la excitación y la estimulación. La subescala final, la urgencia positiva, evalúa la tendencia del individuo a ceder a los impulsos en condiciones de afecto positivo 
alto. Cada elemento de la UPPS-P se midió en una versión de la escala de 4 puntos desde "muy de acuerdo" a "totalmente en desacuerdo". Los valores de confiabilidad reportados por Verdejo-García et al., (2010) para la versión española fueron adecuados para todas las subescalas, urgencia negativa $(, 87)$, falta de premeditación $(, 87)$, falta de perseverancia $(, 79)$, búsqueda de sensaciones $(, 89)$ y urgencia positiva (,93); también fueron adecuados para toda la escala completa $(, 94)$ (Verdejo-García et al., 2010).

\section{Procedimiento}

La investigación se llevó a cabo completamente en la UNC en un ambiente destinado a tal fin, constante a lo largo de todo el estudio. La evaluación a cada participante tuvo una duración de 120 minutos aproximadamente y fue realizada por el responsable de este proyecto y colaboradores entrenados para este fin. Primero, se informó a cada uno de los participantes acerca de las condiciones en que se desarrollaba la investigación, las mismas fueron presentadas por escrito junto con la declaración de consentimiento informado. Este documento debió ser firmado por los participantes. Luego, los participantes recibieron un cuestionario que tenía la finalidad de recabar datos sociodemográficos. A continuación, se procedió a leer y mostrar a través de la pantalla de la computadora las instrucciones de la IGT. Luego se administró la CBT y luego de concluida la misma, se aplicó la WCST. El orden de las pruebas se mantuvo para todos los participantes en igualdad de condiciones, y se estableció según el tiempo de ejecución y cuidando que la realización de una prueba no interfiera en la otra. Una vez finalizadas estas pruebas, se le indicó al participante que contaba con 10 minutos de descanso, que no podía fumar y que tenía agua disponible si lo deseaba. Transcurrido este período de descanso, se aplicó la GDT y luego el cuestionario UPPS-P.

\section{Análisis de Datos}

Para evaluar la evidencia de validez convergente y discriminante los datos fueron analizados siguiendo dos aproximaciones. Por un lado, se analizaron las correlaciones mediante el coeficiente $r$ de Pearson, entre las diferentes variables de todas las pruebas neuropsicológicas utilizadas. Las correlaciones positivas fueron consideradas evidencia de validez convergente, mientras que las correlaciones negativas y la ausencia de correlación fueron consideradas evidencia de validez discriminante. Por otro lado, se realizó un Análisis de Componentes Principales con las puntuaciones de las pruebas de toma de decisiones exclusivamente (Bloques 1 a 5 de la IGT, Puntaje total GDT y Puntaje Total CBT). Se analizó el supuesto de normalidad univariada para todas las variables analizadas mediante la asimetría y la curtosis de la distribución de las variables. En todos los casos los valores de asimetría y curtosis fueron cercanos a cero (Tabachnick \& Fidell, 2007). Para analizar la normalidad multivariada se utilizó la Distancia de Mahalanobis, ningún caso tuvo que ser eliminado ya que no se presentaron valores atípicos según esta medida. Los datos fueron analizados con el software SPSS STATISTICS 19. El nivel de significación para todas las pruebas estadísticas se estableció en $\mathrm{p} \leq, 05$. Se consideró el criterio de Aron, Coups, \& Aron, (2013) para la clasificación de las correlaciones.

\section{Resultados}

\section{Análisis de Correlaciones}

Respecto de la evidencia de validez convergente, se encontraron correlaciones significativas, positivas y débiles, entre las elecciones de mazos desventajosos de la IGT y las elecciones riesgosas de la GDT $(r=, 143)$ y entre las elecciones de mazos ventajosos de la IGT y el porcentaje de respuestas seguras de la GDT $(r=, 153)$. Por otro lado, hubo una correlación significativa positiva y débil entre el porcentaje de elecciones riesgosas GDT y las puntuaciones en la subescala Falta de Premeditación $(r=, 155)$ de la GDT. Por último, en relación a la WCST, el total de respuestas correctas sólo se asoció significativamente con las puntuaciones en la subescala Falta de Premeditación de la UPPS-P, mediante una correlación positiva y débil $(r=, 172)$.

En relación a la evidencia de validez discriminante, en primer lugar, hubo correlación significativa negativa y débil, entre el puntaje total de la IGT y el porcentaje de elecciones riesgosas de la GDT $(r=-, 142)$. Por otro lado, se analizaron las correlaciones de los índices errores perseverativos y errores no perseverativos de la WCST con el resto de las pruebas utilizadas y no se obtuvieron resultados estadísticamente significativos. Respecto de la GDT y la UPPS-P, se encontraron correlaciones significativas negativas y débiles entre el porcentaje de respuestas seguras con la falta de perseverancia $(r=-, 135)$ y con la urgencia negativa $(r=-, 135)$. Para el puntaje total y por bloques de la IGT las correlaciones con los índices de la WCST, CBT y UPPS-P no dieron resultados significativos. Por último, los análisis realizados sobre la puntuación total de la CBT y sus bloques con los demás instrumentos no arrojaron resultados significativos.

\section{Análisis de Componentes Principales}

Además del análisis de las correlaciones entre las puntuaciones de todas las pruebas utilizadas, se utilizó un Análisis Factorial Exploratorio de Componentes Principales con rotación Varimax. La medida KaiserMeyer-Olkin indicó que el análisis fue adecuado $(\mathrm{KMO}=, 798)$ y la prueba de esfericidad de Bartlett fue significativa $\left(\chi^{2}=441,88 p<, 001\right)$ (Pérez \& Medrano, 2010). Para la extracción de los componentes se atendió a los componentes que tuvieran autovalores superiores a 1,00 y al gráfico de sedimentación. Según el primer criterio se debían extraer 3 componentes que dan cuenta del 71,95\% de la varianza, mientras que el gráfico de sedimentación se 
debían extraer 5 componentes que dan cuenta del 91,52\% de la varianza total. En función de esta discrepancia en la cantidad de componentes a extraer, siguiendo las recomendaciones de Costello \& Osbourne, (2005) se probaron soluciones de 3 a 5 componentes. Así, siguiendo las recomendaciones de Hair, Anderson, Tatham, \& Black, (1999) además de atender al criterio estadístico y al criterio gráfico para decidir cuantos componentes extraer se evaluó la significación teórica de cada una de las posibles soluciones. De esta manera se decidió conservar 4 componentes que dan cuenta del $83,88 \%$ de la varianza total (ver Tabla 1). En el primer componente se agruparon los bloques 2 a 5 de la IGT (ensayos 21 a 100) con una varianza explicada del 41,64\%. El segundo componente se compuso exclusivamente del bloque 1 de la IGT (ensayos
1 a 20) con una varianza explicada del $15,77 \%$. El tercer componente se compuso del puntaje total de la GDT, con una varianza explicada del $14,54 \%$. Finalmente, el cuarto componente se compuso del puntaje de la CBT con una varianza del $11,93 \%$. Así, los componentes pueden ser interpretados como "toma de decisiones verídica emocional riesgosa" (Componente 1), "Toma de decisiones verídica emocional ambigua" (Componente 2), "Toma de decisiones verídica no emocional" (Componente 3 ) y "Toma de decisiones centrada en el agente" (Componente 4). Estos resultados van en la línea de lo esperado, es decir, cada tipo de prueba de toma de decisiones abordaría un aspecto distinto de la función y coincide con lo reportado previamente en relación al análisis de las correlaciones entre las puntuaciones de las pruebas.

Tabla 1

Tabla de Análisis de Componentes Principales

\begin{tabular}{lcccr}
\hline & \multicolumn{4}{c}{ Cargas Rotadas (Varimax) } \\
\cline { 2 - 5 } & Factor 1 & Factor 2 & Factor 3 & Factor 4 \\
\hline IGT Bloque 1 &, 127 &, 967 &,- 040 &, 090 \\
IGT Bloque 2 &, 680 &, 288 &, 229 &, 112 \\
IGT Bloque 3 &, 861 &, 144 &, 081 &, 026 \\
IGT Bloque 4 &, 889 &, 032 &,- 017 &, 029 \\
IGT Bloque 5 &, 879 &,- 037 &, 060 &, 029 \\
Puntaje Total GDT &, 055 &,- 034 &, 983 &, 027 \\
Puntaje CBT &, 011 &, 080 & 1,018 &, 835 \\
\hline Autovalores & 2,915 & 1,104 & 14,74 & 1,93 \\
\% Total de Varianza & 41,64 & 15,77 & & 1,93 \\
\hline
\end{tabular}

Nota. IGT=Iowa Gambling Task; GDT=Game of Dice Task; CBT=Cognitive Bias Task

\section{Discusión}

El objetivo principal de este estudio fue analizar la validez convergente y discriminante de un conjunto de pruebas conductuales diseñadas para evaluar la impulsividad rasgo (Escala UPPS-P), la función ejecutiva (FE) (Wisconsin Card Sorting Test) y la TD verídica con y sin componente emocional (Iowa Gambling Task y Game of Dice Task, respectivamente), así como la TD centrada en el agente (Cognitive Bias Task).

Así, en primer lugar, se analizó la puntuación de los participantes en las pruebas de TD y FE atendiendo a la correlación que presentaran entre sí. Adicionalmente, se analizó la relación de estas puntuaciones con una escala de impulsividad rasgo, la UPPS-P.

Los resultados mostraron que la prueba IGT mostró una correlación débil con la prueba GDT. Además, la IGT no correlacionó con las otras pruebas de TD y función ejecutiva, ni con las distintas subescalas de la UPPS-P. Esta falta de correlación entre la IGT y el resto de las pruebas puede interpretarse en términos de validez discriminante. Es decir, se podría considerar que la
IGT podría estar reclutando procesos neurocognitivos distintos de los requeridos por las demás pruebas. En este sentido, la IGT involucra la amígdala en el establecimiento de las primeras reacciones emocionales y las cortezas prefrontales orbitofrontal y ventromedial durante la recuperación de las memorias emocionales (Reimann \& Bechara, 2010).

Por su parte, la correlación débil observada entre la IGT y la GDT, podría entenderse en términos de una convergencia parcial, ya que si bien ambas pruebas evalúan TD, lo hacen reclutando distintas estructuras cerebrales y en escenarios cognitivos diferentes (Schiebener \& Brand, 2015). Brand y colaboradores (2015), sugieren que las $F E$ asociadas con el circuito fronto-estriado tienen un importante papel en la TD bajo condiciones de riesgo objetivo. Así, proponen que el contexto de decisión provee información sobre las reglas de la situación (posibles consecuencias y sus probabilidades asociadas). Esta información se mantiene disponible en la memoria de trabajo. Asimismo, la información de experiencias previas, las estrategias generales de resolución de problemas, y el conocimiento de probabilidades, son recuperados de 
la memoria a largo plazo. Así, las FE son necesarias para categorizar la información relevante, evaluar las opciones disponibles y controlar la aplicación de estrategias.

Asimismo, cabe destacar que, siguiendo el criterio de Podell et al., (2012), la IGT y GDT son pruebas de TD verídica. Es decir, en estas pruebas se pueden identificar unas respuestas más correctas que otras. No obstante, la IGT también puede identificarse como una prueba que evalúa TD bajo riesgo ambiguo. Según lo planteado por la Hipótesis del Marcador Somático (Reimann \& Bechara, 2010), esta prueba evalúa las habilidades del individuo para recuperar los marcadores somáticos de las consecuencias de las elecciones lo cual le permitiría guiar su comportamiento hacia una TD adaptativa. En cambio, la GDT evalúa la TD bajo riesgo seguro. En relación a ello, Schiebener \& Brand, (2015) sostienen que la habilidad para tomar decisiones ventajosas depende de la capacidad para evaluar la probabilidad de éxitos y fracasos por cada elección, lo cual llevaría a una TD más eficiente.

Por otro lado, respecto de la ausencia de correlación entre la IGT y las dimensiones de la UPPS-P, cabe mencionar los resultados de un estudio anterior (Bayard, Raffard, \& Gely-Nargeot, 2011) que tuvo por objetivo evaluar si la impulsividad medida con la UPPS-P predecía la toma de decisiones bajo contextos de riesgo ambiguo (medida con IGT) y riesgo objetivo (medida GDT). En dicho estudio, mientras que la TD bajo riesgo fue predicha por algunas dimensiones de la impulsividad, no se encontraron correlaciones entre las subescalas de la UPPS-P y la IGT. Al respecto, según los autores del estudio mencionado varios antecedentes indican que la evaluación de TD mediante IGT presenta resultados contradictorios y complejos. Un estudio de meta-analítico (Sharma, Markon, \& Clark, 2013) que tuvo como objetivo analizar relaciones ponderadas entre pruebas de auto-reporte y pruebas conductuales de impulsividad, mostró que las pruebas de auto-reporte no correlacionaron consistentemente con las pruebas conductuales entre las que se encontraba la IGT, considerada como una tarea que mide la toma de decisiones impulsivas. En este sentido, los autores explican que la impulsividad, vista como un constructo que atraviesa tanto a las pruebas de auto-reporte como a tareas conductuales no se presentaría como un constructo unitario sino más bien es necesario distinguir entre los rasgos impulsivos y las conductas que conducen a ellos. Considerando el marco teórico que subyace a la TD bajo riego ambiguo (Hipótesis del Marcador Somático; Reimann \& Bechara, (2010) el rendimiento en IGT puede estar influenciado por otros factores como el estado emocional, lo que podría explicar las diferencias en su asociación con otros constructos como la impulsividad (Michelini, Acuña, \& Godoy, 2016). Además, cuando se evalúan participantes controles, la elección de mazos en los últimos bloques de la IGT podría implicar decisiones bajo riesgo objetivo, ya que a lo largo de la prueba, se van haciendo conscientes las reglas de la tarea mediante un aumento en el control atencional del propio comportamiento de TD (Brand et al., 2005).

$\mathrm{Al}$ analizar el rendimiento de los participantes en la GDT, se encontró que las elecciones seguras (las elecciones con probabilidades de ganar mayores o iguales al $50 \%$ ) correlacionaron negativamente con las subescalas Falta de Perseverancia y Urgencia Negativa de la Escala de Impulsividad UPPS-P. Además, las elecciones riesgosas (las elecciones con probabilidades de ganar menores o iguales al 50\%) correlacionaron positivamente con el puntaje en la subescala Falta de Premeditación. Estos resultados son coherentes con estudios anteriores. Por ejemplo Bayard et al., (2011) reportaron que las subescalas Urgencia y Búsqueda de Sensaciones predijeron la tendencia a hacer más elecciones desventajosas bajo riesgo, en las cuales el individuo conoce las probabilidades de ganancia y de pérdida asociadas a su decisión. Estos resultados son esperables si se considera que la Búsqueda de Sensaciones es un constructo intrínsecamente relacionado a la toma de decisiones riesgosas (Para una revisión, véase: Lauriola, Panno, Levin, \& Lejuez, 2014). Además, la Urgencia frecuentemente se asocia a un desempeño bajo en FE, reclutadas en la toma de decisiones bajo riesgo objetivo. En otro estudio donde se evaluó la relación entre la toma de riesgo (medida con la Balloon Analogue Risk Task; BART) y la TD (medida con la IGT) en participantes con alta y baja impulsividad los resultados indicaron que la BART correlacionó con la IGT pero sólo en los últimos bloques y en participantes con baja impulsividad. Al respecto, los autores interpretaron que, a diferencia de los participantes con alta impulsividad, aquellos que presentaron baja impulsividad no mostraron su propensión al riesgo en la IGT hasta que el riesgo no fue aprendido (últimos bloques), lo cual se reflejó en puntajes asociados entre las dos pruebas (BART e IGT). En este sentido y en función de otros antecedentes con resultados similares (Caña, Michelini, Acuña, \& Godoy, 2015), puede considerarse que la impulsividad sólo fue asociada a la TD pero sólo bajo la condición de riesgo objetivo (Brand et al., 2005).

En cuanto a la Wisconsin Card Sorting Test (WCST), los resultados obtenidos son congruentes con lo esperado. Si bien, como se mencionó anteriormente, la evidencia indica que para resolver tareas de TD bajo riesgo objetivo se reclutan las FE, el desempeño en la WCST requiere más bien de habilidades de flexibilidad y control cognitivo, es decir, que el participante actualice el criterio de elección de acuerdo a la retroalimentación que recibe. A diferencia de las pruebas que evalúan TD, en la WCST las reglas son dinámicas, en consecuencia, las contingencias asociadas al castigo o a la recompensa van variando a lo largo de la tarea (Grant \& Berg, 1948). Además, las áreas corticales asociadas al desempeño en la WCST serían más amplias que las reclutadas para 
resolver las tareas de TD. Por ejemplo, en algunos estudios la negatividad asociada a los ensayos erróneos de la WCST tuvo como correlato la activación de áreas corticales prefrontales y áreas corticales parietales vinculadas, precisamente la corteza cingulada anterior (Kuwabara, Mansouri, Buckley, \& Tanaka, 2014).

En el caso de la Cognitive Bias Task (CBT), se encontró que los puntajes de esta prueba no correlacionaron con ninguna de las otras pruebas de TD, WCST y UPPS-P. La ausencia de correlaciones significativas, puede interpretarse en términos de validez discriminante ya que esta prueba se denomina "centrada en el agente", es decir, no pone el acento en lo correcto/incorrecto de las elecciones, sino que se centra en quien decide. En este sentido, resulta relevante analizar si el contexto cognitivo se usa como referencia de las decisiones o no, y si se lo usa, cómo es que se lo usa (Podell, Funk \& Goldberg, 2012).

Por otro lado, el Análisis de Componentes Principales mostró adecuados índices de bondad de ajuste del modelo (Pérez \& Medrano, 2010), con una varianza total explicada del $83,88 \%$. En lo referente a la IGT esta solución coincide con lo reportado por Buelow \& Blaine, (2015) en donde los bloques 2 a 5 (ensayos 21 a 100) se agruparon en un componente denominado "toma de decisiones verídica emocional riesgosa", mientras que el bloque 1 (ensayos 1 a 20) cargó en otro componente denominado "toma de decisiones verídica emocional ambigua". En el mismo sentido Brand, Labudda, \& Markowitsch (2006) también sostienen que la IGT tiene dos etapas de rendimiento diferenciadas, la primera de decisiones ambiguas donde las reglas de decisión no están explícitas y la segunda de decisiones bajo riesgo donde las reglas ya son conocidas o son posibles de intuir.

Por otro lado, la conformación de los otros dos componentes, cada uno con una prueba distinta, obedece a que cada una de ellas mide un aspecto distinto de la toma de decisiones. La GDT, carga en el componente 3 denominado "toma de decisiones verídica no emocional", donde se involucran procesos ejecutivos con reglas de decisión explícitas, configurando una situación de decisión bajo riesgo (Schiebener \& Brand, 2015). Y la CBT, que carga en el componente 4 denominado "toma de decisiones centrada en el agente", evaluando el estilo de la toma de decisiones, es decir, la consideración, o no, del contexto cognitivo al momento de tomar decisiones (Podell et al., 2012). Estos también están en la línea de lo que se encontró al estudiar la convergencia de las puntuaciones mediante el análisis de las correlaciones entre las puntaciones de cada prueba.

Futuros estudios deberían considerar un aumento en el tamaño de la muestra, la inclusión de participantes con distinto nivel educativo y situación laboral, así como una equitativa distribución de hombres y mujeres. Por otro lado, los estudios que tengan como población de interés a jóvenes universitarios deberían asegurar la inclusión de estudiantes de distintas titulaciones. Estas acciones contribuirían a tener un panorama más realista sobre el desempeño en tareas de TD por parte de una población diferente a las típicamente reportadas en este tipo de estudios (ver, por ejemplo: Henrich et al., 2010).

Con todo, este trabajo buscó aportar evidencia empírica sobre los distintos aspectos de la TD que pueden ser evaluados mediante paradigmas conductuales. El análisis de la validez en las puntuaciones de una prueba intenta "ubicar" al constructo en un entramado teórico significativo, dándole coherencia psicológica. Por lo tanto, consideramos que nuestro estudio implica un aporte a la interpretación de las puntuaciones de la evaluación y, en consecuencia, al modo de otorgar significado psicológico a un dato numérico (Carretero-Dios \& Pérez, 2007). En este sentido, las pruebas de TD incluidas en este estudio mostraron evaluar distintos aspectos de la TD de manera ecológica y "adaptable" a culturas diversas. Así, es posible pensar que la inclusión de estas pruebas puede aportar información valiosa sobre la variedad de aspectos teóricos y metodológicos a ser considerados cuando se evalúa $\mathrm{TD}$, ya sea de manera exclusiva o en conjunto con otros dominios cognitivos.

\section{Referencias}

American Educational Research Association, American Psychological Association, \& National Council on Measurement in Education. (2014). Standards for Eudcational and Psychological Testing $\left(1^{\circ}\right)$. Washington, DC: American Educational Research Association.

Aron, A., Coups, E., \& Aron, E. (2013). Statistics for Psychology (6º). New Jersey: Pearson.

Bayard, S., Raffard, S., \& Gely-Nargeot, M. (2011). Do facets of self-reported impulsivity predict decision-making under ambiguity and risk ? Evidence from a community sample. Psychiatry Research, 190(2-3), 322-326. http://doi.org/10.1016/j.psychres.2011.06.013

Bechara, A., Damasio, A., Damasio, H., \& Anderson, S. (1994). Insensitivity to future consequences following damage to human prefrontal cortex. Cognition, 50(1-3), 7-15. Retrieved from http://www.ncbi.nlm.nih.gov/pubmed/8039375

Brand, M., Fujiwara, E., Borsutzky, S., Kalbe, E., Kessler, J., \& Markowitsch, H. J. (2005). Decision-making deficits of korsakoff patients in a new gambling task with explicit rules: associations with executive functions. Neuropsychology, 19(3), 267-277. http://doi.org/10.1037/0894-4105.19.3.267

Brand, M., Labudda, K., \& Markowitsch, H. (2006). Neuropsychological correlates of decision-making in ambiguous and risky situations. Neural Networks, 19(8), 1266-1276. http://doi.org/10.1016/j.neunet.2006.03.001

Brand, M., Recknor, E., Grabenhorst, F., \& Bechara, A. (2007). Decisions under ambiguity and decisions under risk: Correlations with executive functions and comparisons of two different gambling tasks with implicit and explicit rules. Journal of Clinical and Experimental Neuropsychology, $29,86-99$. 
Buelow, M., \& Blaine, A. L. (2015). The Assessment of Risky Decision Making: A Factor Analysis of Performance on the Iowa Gambling Task, Balloon Analogue Risk Task, and Columbia Card Task. Psychological Assessment, (August). http://doi.org/10.1037/a0038622

Caña, M. L., Michelini, Y., Acuña, I., \& Godoy, J. C. (2015). Efectos de la impulsividad y el consumo de alcohol sobre la toma de decisiones en los adolescentes. Health and Addictions/Salud Y Drogas, 15(1), 55-66.

Carretero-Dios, H., \& Pérez, C. (2007). Standards for the development and review of instrumental studies: Considerations about test selection in psychological research. International Journal of Clinical and Health Psychology, 7(3), 863-882.

Costello, A., \& Osbourne, J. (2005). Best practices in exploratory factor analysis: Four recommendations for getting the most from your analysis. Practical Assessment, Research \& Evaluation, 10(7), 1-9. http://doi.org/10.1.1.110.9154

Crivelli, C., Jarillo, S., Russell, J. A., \& Fernández-Dols, J.-M. (2016). Reading emotions from faces in two indigenous societies. Journal of Experimental Psychology: General, 145(7), 830-843. http://doi.org/10.1037/xge0000172

Goldberg, E., Harner, R., Lovell, M., Podell, K., \& Riggio, S. (1994). Cognitive bias, functional cortical geometry and the frontal lobes: laterality, sex and handedness. Journal of Cognitive Neuroscience, 6(3), 276-296.

Goldberg, E., \& Podell, K. (2000). Adaptive decision making, ecological validity, and the frontal lobes. Journal of Clinical and Experimental Neuropsychology, 22(1), 56-68. http://doi.org/10.1076/1380-3395(200002)22:1;1-8;FT056

Grant, D. A., \& Berg, E. A. (1948). A behavioral analysis of degree of reinforcement and ease of shifting to new responses in a Weigl-type card-sorting problem. Journal of Experimental Psychology, 38(4), 404-411.

Hair, J., Anderson, R., Tatham, R., \& Black, W. (1999). Análisis Multivariante (5º. Madrid: Prentice Hall.

Heaton, R., Chelune, G., Talley, J., Kay, G., \& Curtis, G. (1993). Wisconsin Card Sorting Test Manual (1st ed.). Lutz: Psychilogical Assessment Resources.

Henrich, J., Heine, S. J., \& Norenzayan, A. (2010). The weirdest people in the world? The Behavioral and Brain Sciences, 33, 1-23. http://doi. org/10.1017/S0140525X0999152X

Hogan, T. (2004). Pruebas Psicológicas Una introducción práctica (1). México D.F.: El Manual Moderno.

Kuwabara, M., Mansouri, F. A., Buckley, M. J., \& Tanaka, K. (2014). Cognitive control functions of anterior cingulate cortex in macaque monkeys performing a Wisconsin Card Sorting Test analog. The Journal of Neuroscience, 34(22), 7531-7547. http://doi.org/10.1523/ JNEUROSCI.3405-13.2014

Lauriola, M., Panno, A., Levin, I. P., \& Lejuez, C. W. (2014). Individual Differences in Risky Decision Making: A Meta-analysis of Sensation Seeking and Impulsivity with the Balloon Analogue Risk Task. Journal of Behavioral Decision Making, 27(1), 20-36. http://doi.org/10.1002/bdm.1784

Martínez-Selva, J. M., Sánchez-Navarro, J. P., Bechara, A., \& Román, F. (2006). Mecanismos cerebrales de la toma de decisiones. Revista de Neurología, 42(7), 411-418.

Michelini, Y., Acuña, I., \& Godoy, J. C. (2016). Emociones, toma de decisiones y consumo de alcohol en jóvenes universitarios. Suma Psicológica, 23, 42-50. http://doi.org/10.1016/j.sumpsi.2016.01.001

Montero, I., \& León, O. G. (2007). A guide for naming research studies in Psychology. International Journal of Clinical and Health Psychology, $7(3), 847-862$.

Muñiz, J., Elosua, P., \& Hambleton, R. (2013). Directrices para la traducción y adaptación de los tests: Segunda edición. Psicothema, 25(2), 151-157. http://doi.org/10.7334/psicothema2013.24

Pérez, E., \& Medrano, L. (2010). Análisis Factorial Exploratorio: Bases Conceptuales y Metodológicas. Revista Argentina de Ciencias Del Comportamiento, 2(1889), 58-66.

Podell, K., Funk, B., \& Goldberg, E. (2012). Agent-Centered Decision Making in normal and abnormal cognition. Revista Argentina de Ciencias Del Comportamiento, 4(2), 32-42.

Reimann, M., \& Bechara, A. (2010). The somatic marker framework as a neurological theory of decision-making: Review, conceptual comparisons, and future neuroeconomics research. Journal of Economic Psychology, 31 (5), 767-776. http://doi.org/10.1016/j.joep.2010.03.002

Schiebener, J., \& Brand, M. (2015). Decision Making Under Objective Risk Conditions - a Review of Cognitive and Emotional Correlates, Strategies, Feedback Processing, and External Influences. Neuropsychology Review, 25(2), 171-198. http://doi.org/10.1007/s11065-015-9285-x

Sharma, L., Markon, K. E., \& Clark, L. A. (2013). Toward a theory of distinct types of "impulsive" behaviors: A meta-analysis of self-report and behavioral measures. Psychological Bulletin, 140(2), 374-408. http://doi.org/http://dx.doi.org.sire.ub.edu/10.1037/a0034418

Tabachnick, B., \& Fidell, L. (2007). Using Multivariate Statistics (5th ed.). Boston: Pearson Education.

Verdejo-García, A., Lozano, Ó., Moya, M., Alcázar, M. Á., \& Pérez-García, M. (2010). Psychometric properties of a Spanish version of the UPPS-P impulsive behavior scale: reliability, validity and association with trait and cognitive impulsivity. Journal of Personality Assessment, 92(1), 70-77. http://doi.org/10.1080/00223890903382369

Verdejo-García, A., Vilar-López, R., Pérez-García, M., Podell, K., Goldberg, E., Vilar-Lopez, R., ... Goldberg, E. (2006). Altered adaptive but not veridical decision-making in substance dependent individuals. Journal of the International Neuropsychological Society, 12(1), 90-99. http://doi.org/10.1017/S1355617706060127

Wood, P. K., Sher, K., \& Rutledge, P. C. (2007). College Student Alcohol Consumption, Day of the Week, and Class Schedule. Alcoholism: Clinical and Experimental Research, 31(7), 1195-1207. http://doi.org/10.1111/j.1530-0277.2007.00402.x

recebido em outubro de 2016 aprovado em julho de 2017

\section{Sobre os autores}

Ignacio Acuña es Licenciado y Doctor en Psicología por la Univesidad Nacional de Córdoba. Becario Postdoctoral en el IIPSI-UNCCONICET (Argentina) y Profesor Asistente en la Universidad Nacional de Córdoba.

Yanina Michelini es Licenciada en Psicología por la Universidad Nacional de Córdoba. Becaria Doctoral en el IIPSI-UNC-CONICET (Argentina) y Profesora Asistente en la Universidad Nacional de Córdoba.

Juan Ignacio Guzmán es Licenciado en Psicología por la Universidad Nacional de Córdoba. Becario CIN en el IIPSI-UNCCONICET (Argentina).

Juan Carlos Godoy es Profesor, Licenciado y Doctor en Psicología por la Universidad Nacional de Córdoba. Investigador Adjunto del CONICET en el IIPSI-UNC-CONICET (Argentina) y Profesor Adjunto en la Universidad Nacional de Córdoba. 